\title{
Visualization of Lenticulostriate Arteries on CT Angiography Using Ultra-High-Resolution CT Compared with Conventional-Detector CT
}

\author{
(D) K. Murayama, (D) S. Suzuki, (DH. Nagata, (D). Oda, (DI. Nakahara, (DK. Katada, (DK. Fujii, and (D) H. Toyama
}

\begin{abstract}
BACKGROUND AND PURPOSE: The newly developed ultra-high-resolution CT is equipped with a 0.25 -mm detector, which has one-half the conventional section thickness, one-half the in-plane detector element width, and one-half the reconstructed pixel width compared with conventional-detector CT. Thus, the ultra-high-resolution CT scanner should provide better image quality for microvasculature than the conventional-detector CT scanners. This study aimed to determine whether ultra-high-resolution CT produces superior-quality images of the lenticulostriate arteries compared with conventional-detector CT.
\end{abstract}

MATERIALS AND METHODS: From February 2017 to June 2017, thirteen patients with aneurysms (4 men, 9 women; mean age, 61.2 years) who underwent head CTA with both ultra-high-resolution CT and conventional-detector CT were enrolled. Two boardcertified radiologists determined the number of all lenticulostriate arteries on the CTA coronal images of the MCA M1 segment reconstructed from 512 matrixes on conventional-detector CT and 1024 matrixes on ultra-high-resolution CT.

RESULTS: There were statistically more lenticulostriate arteries identified on ultra-high-resolution CT (average, $2.85 \pm 0.83 ; 95 \% \mathrm{Cl}$, 2.509-3.183) than on conventional-detector CT (average, $2.17 \pm 0.76 ; 95 \% \mathrm{Cl}$, 1.866-2.480) $(P=.009)$ in 16 of the total $26 \mathrm{MCA} \mathrm{Ml}$ segments.

CONCLUSIONS: Improvements in lenticulostriate artery visualization were the result of the combined package of the ultra-high-resolution CT scanner plus the ultra-high-resolution scanning protocol, which includes higher radiation doses with lower than the national diagnostic reference levels and stronger adaptive iterative dose-reduction processing. This package for ultra-high-resolution $\mathrm{CT}$ is a simple, noninvasive, and easily accessible method to evaluate microvasculature such as the lenticulostriate arteries.

ABBREVIATIONS: AIDR = adaptive iterative dose reduction; $\mathrm{C}-\mathrm{CT}=$ conventional-detector $\mathrm{CT}$; $\mathrm{C}-\mathrm{CTA}=$ conventional $\mathrm{CTA}$; $\mathrm{CTDI}_{\mathrm{vol}}=$ volume $_{\mathrm{CT}}$ dose index; LSA = lenticulostriate artery; UHR = ultra-high-resolution

$\mathbf{T}$ he lenticulostriate arteries (LSAs) are perforating arteries that originate in the MCA and supply the basal ganglia. Accurately assessing the variation and structure of the LSAs reduces the likelihood of postoperative complications when performing resections of insular gliomas and when clipping or

Received November 2, 2018; accepted after revision November 12, 2019.

From the Joint Research Laboratory of Advanced Medical Imaging (K.M.), Departments of Radiology (S.S., H.N., K.K., H.T.) and Comprehensive Strokology (J.O., I.N.), Fujita Health University, Aichi, Japan; and Canon Medical Systems (K.F.) Otawara, Japan.

This work was supported by Grants-in-Aid for Scientific Research (KAKENHI) 18 K07694.

Paper previously presented at: Annual Meeting of the Japan Radiological Society, April 12-15, 2018; Yokohama, Japan.

Please address correspondence to Kazuhiro Murayama, MD, PhD, Joint Research Laboratory of Advanced Medical Imaging, Fujita Health University, 1-98

Dengakugakubo, Kutsukake-cho Toyoake, Aichi, 470-1101, Japan;

e-mail: kmura@fujita-hu.ac.jp

- Indicates open access to non-subscribers at www.ajnr.org

http://dx.doi.org/10.3174/ajnr.A6377 coiling MCA M1-2 segment aneurysms. ${ }^{1,2}$ It is also clinically important to evaluate the features of the LSAs in patients with symptomatic intracranial atherosclerotic stenosis to determine the best therapeutic strategy. ${ }^{3}$ However, although conventionaldetector CT (C-CT), which uses a detector with a range of $0.5 \mathrm{~mm}$, has been used to image the proximal side of the LSA, it is not easy to image the entire LSA from the proximal-to-distal side using C-CT.

In 2005, Canon Medical Systems and the National Cancer Center, Japan, jointly developed a prototype ultra-high-resolution CT system (UHR-CT; TSX-304R; Canon Medical Systems, Otawara, Japan). The UHR-CT was equipped with a $0.25-\mathrm{mm}$ detector, which has one-half the conventional section thickness, one-half the in-plane detector element width, and one-half the reconstructed pixel width compared with the C-CT. Kakinuma et $\mathrm{al}^{5}$ reported that the prototype UHR-CT scanner had significantly better image quality for lung nodules than the C-CT scanners. Clinical studies that have tested phantoms using the 
production version of the UHR-CT (Aquilion Precision; Canon Medical Systems) have reported that a higher image quality could be obtained compared with C-CT. ${ }^{6,7}$ Another such study that imaged the artery of Adamkiewicz reported improved images. ${ }^{8}$ On the basis of these findings, we predicted that UHRCTA will have improved imaging quality of the LSAs that exceeds that of conventional CTA (C-CTA). Therefore, the goal of this study was to investigate whether UHR-CTA produced superior images of the LSAs compared with C-CTA.

\section{MATERIALS AND METHODS Subjects}

Our institutional review board (Fujita Health University) approved this opt-out research design. Opt-out opportunities were provided to all participants included in this retrospective study. Forty-two consecutive patients with intracranial aneurysms who underwent intracranial UHR-CTA between February 2017 and June 2017 and who underwent C-CTA using 320-row detector CT during a follow-up study (14-373 days later; mean interval, 224 days) were selected. Twenty-nine patients were excluded for the following reasons: unavailable datasets, the scanning parameters differing from those defined in the protocol, insufficient scanning range for evaluating the LSAs, and enhanced adaptive iterative dose reduction 3D (AIDR 3D; Canon Medical Systems $)^{9,10}$ not used for the denoising procedure. Therefore, 13 patients ( 4 men and 9 women; age range, 35-74 years; mean age, 61.2 years) with 26 bilateral MCA M1 segments were enrolled in this study.

\section{CTA Protocols for UHR-CT and C-CT}

Contrast-enhanced volume data for UHR-CTA were acquired with a 160-detector row UHR-CT scanner (Aquilion Precision) using helical scanning. The UHR-CT scanner used in this study is a medical device with certification as a CT scanner that is safe for clinical use. The other scan parameters were as follows: section thickness of $0.25 \mathrm{~mm}$, tube voltage of $120 \mathrm{kV}$, automatic exposure control tube current SD of 5, pitch factor of 0.625 , reconstructed FOV size of $190-240 \mathrm{~mm}$, and gantry rotation speed of $1 \mathrm{~s} /$ rotation. Contrast-enhanced volume data for C-CTA were acquired with a 320-row detector CT scanner (Aquilion ONE; Canon Medical Systems) using nonhelical scanning. The other scan parameters were as follows: section thickness of $0.5 \mathrm{~mm}$, tube voltage of $120 \mathrm{kV}$, automatic exposure control tube current SD of 3-5, pitch factor of 0.625 , reconstructed FOV size of $180-240 \mathrm{~mm}$, and gantry rotation speed of 1 s/rotation. The contrast medium volume was calculated on the basis of each patient's body weight, and $250 \mathrm{mg} \mathrm{I} / \mathrm{kg}$ of iopamidol (Iopamiron 370; Bayer Healthcare, Osaka, Japan) was injected intravenously as a bolus for a fixed infusion duration of 10 seconds, followed by an intravenous bolus injection of $30 \mathrm{~mL}$ of physiologic saline solution at the same rate as the contrast medium.

\section{Image Postprocessing and Data Analysis}

The CTA coronal MIP images of 20-mm thick MCA M1 segments were reconstructed from a pixel matrix of $512 \times 512$ for C-CTA with a $0.5-\mathrm{mm}$ thickness and a pixel matrix of $1024 \times$ 1024 for UHR-CTA with a $0.25-\mathrm{mm}$ thickness with a strong
AIDR 3D. C-CTA was reconstructed with a weak AIDR 3D using the analysis software installed in the CT console. Previous studies evaluating the LSAs on MR imaging and CTA determined the number of LSA branches within $5 \mathrm{~mm}$ from the MCA and the number of branches beyond $5 \mathrm{~mm} .{ }^{11,12}$ The number of LSAs, defined as those longer than $5 \mathrm{~mm}$, were analyzed. ${ }^{12}$ Two boardcertified radiologists (K.M. with 14 years of experience; S.S. with 14 years of experience) participated as observers. A standard window level and width were applied during reader observations. The observers were allowed to freely vary window levels and widths.

\section{Statistical Analysis}

We compared the mean visible number of LSAs recorded by 2 blinded and independent observers between the C-CTA and UHR-CTA images. A Wilcoxon matched-pairs signed rank test was used to calculate $P$ values for comparisons. For all statistical analyses, a $P$ value of $<.05$ indicated a statistically significant between-group difference. The statistical analyses were performed using commercially available statistical software (GraphPad Prism software, Version 6, GraphPad Software, San Diego, California; BellCurve for Excel, Version 2.11, Social Survey Research Information, Tokyo, Japan).

\section{Radiation Dose}

The estimated volume CT dose index $\left(\mathrm{CTDI}_{\mathrm{vol}}\right)$ for CTA displayed on the CT scanner console was recorded for each patient. The estimated dose-length product was calculated as $\mathrm{CTDI}_{\mathrm{vol}} \times$ Scan Length. The radiation dose was also compared against the diagnostic reference level of the Japan Network for Research and Information on Medical Exposure (J-RIME) (http://www.radher. jp/J-RIME/report/DRLhoukokusyoEng.pdf). The J-RIME is the network used to collect data related to medical exposure and to construct a framework within Japan for appropriate medical radiation doses. The J-RIME suggested that national diagnostic reference levels established in 2015 for routine brain CT examination were $85 \mathrm{mGy}\left(\mathrm{CTDI}_{\mathrm{vol}}\right)$ and $1350 \mathrm{mGy} \times \mathrm{cm}$ (dose-length product).

\section{RESULTS}

The number of LSAs determined by the 2 observers is summarized in Fig 1. There were statistically more LSAs identified on UHR-CT (average, $2.85 \pm 0.83$; 95\% CI, 2.509-3.183) than on CCT (average, $2.17 \pm 0.76$; 95\% CI, 1.866-2.480) $(P=.009$ ). There were also more LSAs identified on UHR-CT than on C-CT in 16 of the total 26 MCA M1 segments. Figures 2 and 3 show representative images that clearly show bilateral LSAs from the proximal-to-distal tip in UHR-CTA compared with LSAs imaged by C-CTA, respectively.

The CTDI ${ }_{\text {vol }}$ obtained with C-CT was 9.7-37.7 mGy (mean, $27.2 \mathrm{mGy}$ ), whereas it was 44.7-63.1 mGy (mean, 53.8 mGy) with UHR-CT. The estimated dose-length product range was calculated as $\mathrm{CTDI}_{\mathrm{vol}} \times$ Scan Length, which was determined as 124.9-488.1 mGy $\times \mathrm{cm}$ (mean, $266.5 \mathrm{mGy} \times \mathrm{cm})$ with C-CT and 685.8-942.5 $\mathrm{mGy} \times \mathrm{cm}$ (mean, $740.8 \mathrm{mGy} \times \mathrm{cm})$ with UHR-CT. The radiation doses for both C-CT and U-HRCT 


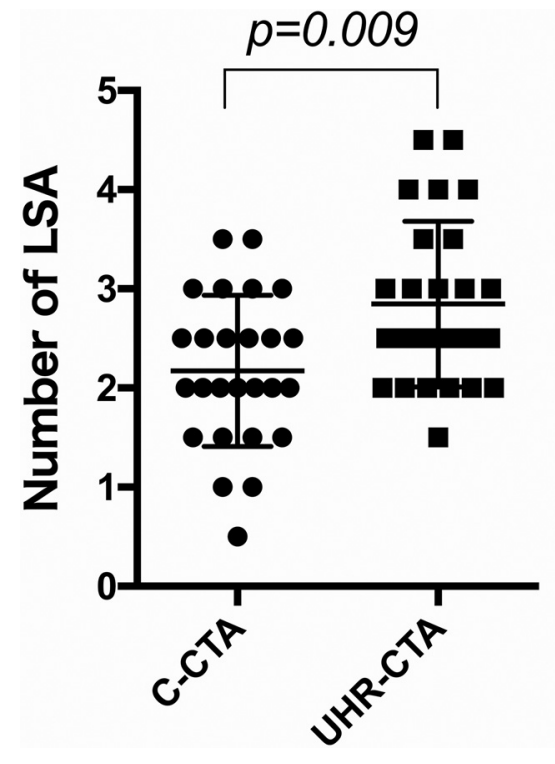

FIG 1. Plots of the number of LSAs between C-CTA and UHR-CTA. UHR-CTA identified more MCA perforators than C-CTA. were still lower than the diagnostic reference levels according to J-RIME.

\section{DISCUSSION}

Our results suggest that UHR-CT provides higher quality images of intracranial CTA than C-CT because of improved spatial resolution and partial volume effect. UHR-CTA is a simple, noninvasive, and easily accessible method to evaluate microvasculature such as the LSAs.

LSA imaging has various important clinical roles. Surgically, it is important to accurately assess the anatomic structures of the LSAs before insular glioma surgery and coiling and clipping of MCA aneurysms. ${ }^{13}$ Additionally, LSA imaging is an important step toward reducing postoperative complications. ${ }^{9}$ Patients with hypertension have significantly fewer LSAs compared with healthy volunteers. ${ }^{14}$ It is also important to evaluate LSA features in patients with symptomatic intracranial atherosclerotic stenosis. ${ }^{3}$ Investigations using flow-sensitive black-blood MRA with MR imaging have confirmed that it is superior for visualizing LSA branches in patients with stroke. ${ }^{11,15}$ Other studies using 7T MR imaging have reported advanced noninvasive methods for visualization of the cerebral microvasculature, including the LSAs. ${ }^{16,17}$ However, it is not easy to image the LSA when using TOF-MRA with conventional $1.5 \mathrm{~T}$ MR imaging systems, which is the most prevalent technique currently available. Digital subtraction angiography is considered the criterion standard of cerebral vascular imaging, and 3D rotational angiography on an x-ray angiographic system is useful for identifying an important anatomic relationship between the MCA trunk and LSAs; ${ }^{18}$ however, it is an invasive procedure and has some risk of complications. Therefore, noninvasive CTA or MR imaging is necessary for imaging microvasculature, such as LSAs.

The world's first UHR-CT scanner is equipped with a $0.25-\mathrm{mm}$ detector of the physical size scaled back to the isocenter that has pixels onequarter the size of those of the C-CT scanner. ${ }^{5}$ Kakinuma et $\mathrm{al}^{5}$ reported that the prototype UHR-CT scanner had a significantly better image quality for lung nodules than conventional high-resolution CT scanners. As has been observed in previous phantom tests and clinical studies of other organs, ${ }^{6-8}$ the results of our investigation showed that UHR-CTA improved the image quality of the LSAs compared with C-CTA (Figs 2 and 3). This difference is likely
FIG 3. A 74-year-old woman with bilateral MCA aneurysms. A, C-CTA of a coronal partial MIP $(20 \mathrm{~mm})$ image. B, UHR-CTA of a coronal partial MIP $(20 \mathrm{~mm})$ image. UHR-CTA shows bilateral LSAs more clearly (arrows), and it allows accurate assessment of the variation and structure of the LSAs compared with C-CTA. 
attributable to the spatial resolution of the UHR-CT and its associated reduction in the partial volume effects for the following reasons: First, UHR-CT provides significantly higher quality images of intracranial CTA using detectors with half the thickness in the $\mathrm{z}$-axon direction $(0.25 \mathrm{~mm} \times 160$ detectors) and twice as many channels in the $\mathrm{x}$ - and $\mathrm{y}$-axon directions (1792 channels) on the super-high-resolution dataacquisition mode compared with C-CT. Second, the focal spot size of the UHR $\mathrm{x}$-ray tubes $(0.4 \times 0.5 \mathrm{~mm})$ is smaller than that of C-CT $\mathrm{x}$-ray tubes $(0.9 \times 0.8 \mathrm{~mm})$. Finally, UHR-CTA is reconstructed by a higher resolution matrix $(1024 \times 1024)$ compared with C-CTA (matrix size, $512 \times 512$ ). On the basis of these factors, UHR-CT appears to provide significantly higher quality images of intracranial CTA than C-CT. Thus, UHR-CTA allows us to accurately assess the variation and structure of LSAs.

Differences in the imaging quality of C-CTA and UHR-CTA include the difference between helical and nonhelical scanning in addition to the spatial resolution as mentioned above; these differences influence the image quality of contrast-enhanced images. ${ }^{19-21}$ Because UHR-CT must use helical scanning, UHRCTA image homogeneity with helical scans can be inferior to that of C-CTA with nonhelical scanning. ${ }^{22}$ Furthermore, it may be difficult to obtain images with optimal contrast-enhanced timing because UHR-CTA requires a longer scanning time. UHR-CTA is significantly superior to C-CTA for imaging the LSAs despite these disadvantages; this difference implies the greater advantages of UHR-CTA compared with C-CTA. In some cases, the number of LSAs on UHR-CT was the same or fewer than on C-CT for the following reasons: CTA scanning on UHR-CT must use the super-high-resolution mode, which has the smallest size of scan focus. The super-high-resolution mode has the technical upper limit of radiation output power; therefore, all cases showed nearly the same values for CTDI and dose-length product. As a result, we predicted that this occurred when the radiation doses were not sufficient for UHR-CT imaging to visualize LSAs in some cases.

This study has several limitations. First, a small number of subjects were included in this retrospective analysis; future prospective studies should include more subjects. Second, the scan protocols such as the FOV, reconstruction, and AIDR 3D were not the same between UHR-CTA and C-CTA. It was difficult to scan all CTA images using completely identical conditions between UHR-CTA and C-CTA because these scan protocols were optimized individually for the equipment for each C-CT and UHR-CT. Third, C-CT scans were obtained at a radiation dose range (9.7-37.7 $\mathrm{mGy} \mathrm{CTDI}_{\mathrm{vol}}$ ) that is much lower than the 85-mGy diagnostic reference level. There was a possibility that LSAs were poorly visualized in C-CT simply because of the lower dose and correspondingly higher image noise levels. The comparisons between C-CT and UHR-CT should be performed at the same radiation dose. Fourth, UHR-CTA was compared with C-CTA, with C-CTA performed an average of 224 days after the initial CTA. We detected no new lesions between the scans on CT; however, the visualization of the LSAs on the follow-up scans could be affected by pathologic changes or micromorphologic changes.

\section{CONCLUSIONS}

Improvement in LSA visualization was the result of the combined package of the UHR-CT scanner plus the UHR scanning protocol, which includes a higher radiation dose with lower than the national diagnostic reference levels and stronger AIDR processing. This package for UHR-CT is a simple, noninvasive, and easily accessible method to evaluate microvasculature such as the LSAs.

Disclosures: Kazuhiro Murayama-RELATED: Grant: Grants-in-Aid for Scientific Research (KAKENHI).* Ichiro Nakahara-UNRELATED: Consultancy: Kaneka Medix (Japan), Terumo (Japan), Nipro Corporation (Japan). Kazuhiro KatadaRELATED: Consultancy: Canon Medical Systems; Grants/Grants Pending: Canon Medical Systems.* Kenji Fujii-RELATED: Employment: Canon Medical Systems. *Money paid to the institution.

\section{ACKNOWLEDGMENT}

The authors would like to thank Maruzen-Yushodo Co Ltd (https://kw.maruzen.co.jp/kousei-honyaku/) for the English language editing.

\section{REFERENCES}

1. Steno A, Jezberova M, Holly V, et al. Visualization of lenticulostriate arteries during insular low-grade glioma surgeries by navigated 3D ultrasound power Doppler: technical note. J Neurosurg 2016; 125:1016-23 CrossRef Medline

2. Moshel YA, Marcus JD, Parker EC, et al. Resection of insular gliomas: the importance of lenticulostriate artery position. J Neurosurg 2008;109:825-34 CrossRef Medline

3. Wang M, Wu F, Yang Y, et al. Quantitative assessment of symptomatic intracranial atherosclerosis and lenticulostriate arteries in recent stroke patients using whole-brain high-resolution cardiovascular magnetic resonance imaging. $J$ Cardiovasc Magn Reson 2018;20:35 CrossRef Medline

4. Gotoh K, Okada T, Satogami N, et al. Evaluation of CT angiography for visualisation of the lenticulostriate artery: difference between normotensive and hypertensive patients. Br J Radiology 2012;85: e1004-08 CrossRef Medline

5. Kakinuma R, Moriyama N, Muramatsu Y, et al. Ultra-high-resolution computed tomography of the lung: image quality of a prototype scanner. PLoS One 2015;10:e0137165 CrossRef Medline

6. Yanagawa $\mathrm{M}$, Hata A, Honda $\mathrm{O}$, et al. Subjective and objective comparisons of image quality between ultra-high-resolution CT and conventional area detector $\mathrm{CT}$ in phantoms and cadaveric human lungs. Eur Radiol 2018;28:5060-68 CrossRef Medline

7. Hata A, Yanagawa M, Honda O, et al. Effect of matrix size on the image quality of ultra-high-resolution $\mathrm{CT}$ of the lung: comparison of $512 \times 512,1024 \times 1024$, and $2048 \times 2048$. Acad Radiol 2018;25:869-76 CrossRef Medline

8. Yoshioka K, Tanaka R, Takagi H, et al. Ultra-high-resolution CT angiography of the artery of Adamkiewicz: a feasibility study. Neuroradiology 2018;60:109-15 CrossRef Medline

9. Matsuki M, Murakami T, Juri $\mathrm{H}$, et al. Impact of adaptive iterative dose reduction (AIDR) 3D on low-dose abdominal CT: comparison with routine-dose CT using filtered back projection. Acta Radiol 2013;54:869-75 CrossRef Medline

10. Feger S, Rief M, Zimmermann E, et al. The impact of different levels of adaptive iterative dose reduction $3 \mathrm{D}$ on image quality of 320row coronary CT angiography: a clinical trial. PLoS One 2015;10: e0125943 CrossRef Medline

11. Okuchi S, Okada T, Ihara M, et al. Visualization of lenticulostriate arteries by flow-sensitive black-blood MR angiography on a $1.5 \mathrm{~T}$ MRI system: a comparative study between subjects with and without stroke. AJNR Am J Neuroradiol 2013;34:780-84 CrossRef Medline 
12. Gotoh K, Okada T, Miki Y, et al. Visualization of the lenticulostriate artery with flow-sensitive black-blood acquisition in comparison with time-of-flight MR angiography. J Magn Reson Imaging 2009; 29:65-69 CrossRef Medline

13. Akashi T, Taoka T, Ochi T, et al. Branching pattern of lenticulostriate arteries observed by MR angiography at 3.0 T. Jpn J Radiol 2012;30:331-35 CrossRef Medline

14. Chen YC, Li MH, Li YH, et al. Analysis of correlation between the number of lenticulostriate arteries and hypertension based on high-resolution MR angiography findings. AJNR Am J Neuroradiol 2011;32:1899-1903 CrossRef Medline

15. Okuchi S, Okada T, Fujimoto K, et al. Visualization of lenticulostriate arteries at 3T: optimization of slice-selective off-resonance sinc pulse-prepared TOF-MRA and its comparison with flow-sensitive black-blood MRA. Acad Radiol 2014;21:812-16 CrossRef Medline

16. Cho ZH, Kang CK, Han JY, et al. Observation of the lenticulostriate arteries in the human brain in vivo using 7.0T MR angiography. Stroke 2008;39:1604-06 CrossRef Medline

17. Yashiro S, Kameda H, Chida A, et al. Evaluation of lenticulostriate arteries changes by $7 \mathrm{~T}$ magnetic resonance angiography in type 2 diabetes. J Atheroscler Thromb 2018;25:1067-75 CrossRef Medline
18. Park JC, Shim JH, Lee DH, et al. Three-dimensional angiographic evaluation of middle cerebral artery trunk aneurysms: demonstration of the close relationship between the early frontal cortical branches and lateral lenticulostriate arteries. World Neurosurg 2016;91:383-89 CrossRef Medline

19. Saake M, Goelitz P, Struffert T, et al. Comparison of conventional CTA and volume perfusion CTA in evaluation of cerebral arterial vasculature in acute stroke. AJNR Am J Neuroradiol 2012;33:206873 CrossRef Medline

20. Honda $\mathrm{O}$, Yanagawa $\mathrm{M}$, Hata $\mathrm{A}$, et al. Influence of gantry rotation time and scan mode on image quality in ultra-high-resolution CT system. Eur J Radiol 2018;103:71-75 CrossRef Medline

21. Jeon SK, Choi YH, Cheon JE, et al. Unenhanced 320-row multidetector computed tomography of the brain in children: comparison of image quality and radiation dose among wide-volume, one-shot volume, and helical scan modes. Pediatr Radiol 2018;48:594-601 CrossRef Medline

22. Honda $\mathrm{O}$, Takenaka $\mathrm{D}$, Matsuki $\mathrm{M}$, et al. Image quality of 320-detector row wide-volume computed tomography with diffuse lung diseases: comparison with 64-detector row helical CT. J Comput Assist Tomogr 2012;36:505-11 CrossRef Medline 